\title{
EFECTIVIDAD DE UN PROGRAMA EDUCATIVO SOBRE AUTOCUIDADO EN LA PREVENCIÓN DE COMPLICACIONES DE MIEMBROS INFERIORES EN PACIENTES CON DIABETES MELLITUS HOSPITAL SANTA MARIA DEL SOCORRO DE ICA 2014- 2015
}

Effectiveness of an educational program on self-care in the prevention of complications of lower members in patients with diabetes mellitus Hospital Santa Maria Socorro of Ica 2014-2015

Flora Martha Huisacayna Díaz 1, a, c, e ,Yolanda Aguilar Semino de Flores 3, a , Ana Cecilia Flores García4, a, d, Linda Karim Ninahuaman Huisacayna ${ }^{2, b}$, Redu Yupanqui ${ }^{1, f}$

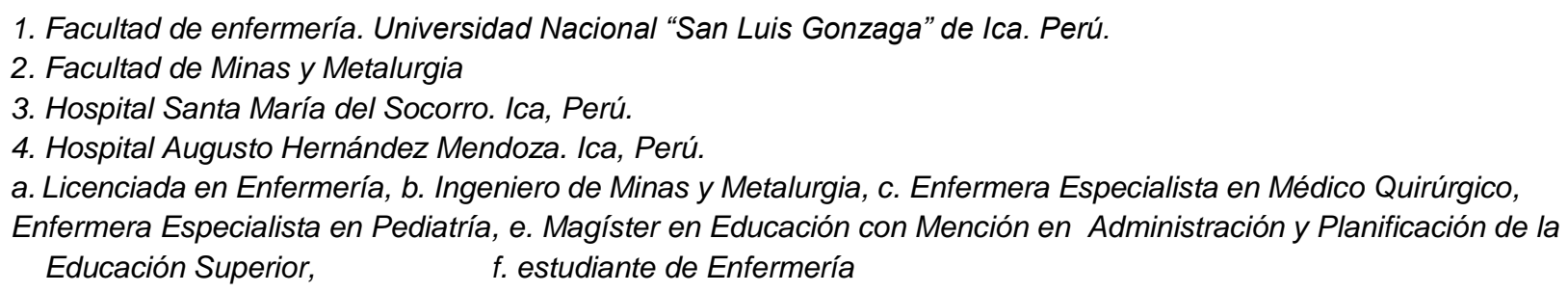

\section{RESUMEN}

Objetivo: Evaluar la efectividad del programa educativo sobre autocuidado en miembros inferiores de los pacientes con Diabetes mellitus que acuden al hospital Santa María del Socorro de Ica Setiembre 2014- Agosto 2015. Material y métodos: Se trata de una investigación, del tipo aplicativa explicativa longitudinal, con diseño Cuasi experimental con grupo control y grupo experimental La muestra estuvo conformada por 100 personas con diagnóstico de Diabetes Mellitus tipo 2 ; 50 pacientes para grupo control y 50 para grupo experimental que reunieron los criterios de selección y, consentimiento informado .Se utilizó como técnica la encuesta y como instrumento un cuestionario de 10 preguntas de conocimiento sobre pie diabético y 43 Ítems sobre autocuidado de los miembros inferiores La validez del instrumento se determinó mediante juicios de expertos y la confiabilidad ,Alfa de Cronbach fue de 0,79. RESULTADOS después de aplicado el programa educativo sobre complicaciones de miembros inferiores a pacientes con diabetes Mellitus tipo 2, se incrementó el nivel de conocimiento en el grupo experimental de $30,4 \%$ a $71,6 \%$ y en el nivel de autocuidado de $30,64 \%$ a 75,88\%; el grupo control permaneció con los mismos niveles bajos, posterior se dio el mismo programa educativo al grupo control

Palabras claves: Programa educativo, Diabetes Mellitus, prevención, miembros inferiores

\section{SUMMARY}

Objective : evaluate the efficiency of the educational program on car taken care in low members of the patients with Diabetes mellitus that come to the hospital Santa Maria of Ica Material and Methods: It is a question of an investigation, of the type applicative explanatory longitudinal, with design experimental Cuasi with group control and experimental group The sample was shaped by 100 persons by diagnosis of Diabetes Mellitus type 2, 50 patients for group control and 50 for experimental group that the criteria of selection assembled and, informed assent followed by an educational program. For the compilation of information the survey was in use as technology and since I orchestrate a questionnaire of 10 questions of knowledge on diabetic foot and 43 Articles on car taken care of the low members The knowledge, habits of elegant car were deficient before the educational intervention, later the change was highly significant The validity decided by means of experts' judgments and the reliability, Cronbach Alpha was of 0.79 Results after applied the educational program on complications of limbs in patients with diabetes Mellitus type 2, increased the level of knowledge in the experimental group of $30.4 \%$ to $71.6 \%$ and at the level of self-care $30.64 \%$ a $75.88 \%$; the group control remained at the same low levels, later the same educational program was given to the control group

Key words: educational Program, Diabetes Mellitus, prevention, low members. 


\section{INTRODUCCION}

La diabetes mellitus es una patología que ha adquirido características epidémicas a nivel mundial, a tal grado que la Organización Mundial de la Salud (OMS), refiere que el número de personas ha aumentado de 108 millones en 1980 a 422 millones en 1014, la prevalencia de la diabetes en adultos (mayores de 18 años) ha aumentado con mayor rapidez en los países de ingreso mediano y bajo La diabetes es una importante causa de la ceguera, insuficiencia renal infarto del miocardio accidente cerebro vascular y amputaciones de miembros inferiores, se estima que en 2012 la diabetes fue la causa directa de 1,5 millones de muertes y que otros 2,2 millones eran atribuibles a la hiperglicemia (1) .

El pie diabético es una de las complicaciones degenerativas, que ocasiona invalidez presentándose en pacientes con una evolución larga y un nivel de autocuidado bajo es considerado como una de las tres primeras causas de mortalidad en los pacientes con diabetes Se define el Pie diabético, como una alteración clínica de base etiopatogenia neuropatía e inducida por la hiperglucemia mantenida, en la que con o sin coexistencia de isquemia, y previo desencadenante traumático, produce lesión y/o ulceración del pie (2). El pie del paciente diabético es muy sensible a todas formas de traumatismos: el talón y las prominencias óseas resultan especialmente vulnerables. Los daños a los nervios periféricos de los pies provocan trastornos sensoriales, úlceras de la planta del pie, atrofia de la piel, etc. y debido a la oclusión de las arterias que llevan sangre a los pies se puede producir gangrena Es frecuente en los pacientes diabéticos que las lesiones propias del denominado pie diabético trascurran sin dolor, debido a lo cual se suele agravar la lesión antes de que el paciente pida ayuda especializada (2). La prevalencia de úlceras de pie diabético en los pies desarrollados varía según el sexo, edad y población desde el $4 \%$ hasta el $10 \%$ se ha establecido una incidencia correspondiente del 2,2 al $5,9 \%$. Se ha calculado que al menos un $15 \%$ de los diabéticos padecerá durante su vida ulceraciones en el pie, igualmente se estima alrededor de un $85 \%$ de los diabéticos que sufren amputaciones previamente han padecido una úlcera La prevalencia estimada de neuropatías periféricas, factor de riesgo prevalerte para el desarrollo de ulceras oscila entre el 30 y el $70 \%$.

La prevalecía de enfermedad vascular periférica en diabéticos se ha calculado que oscila del 10-20\% (Consenso Internacional sobre Pie Diabético 2001). Podemos afirmar que las úlceras neuropatías son de igual frecuencia en hombres que en mujeres y que aparecen generalmente en diabéticos de larga evolución (2). La enfermera dentro de las acciones preventivas promocional cumple un rol muy importante en el cuidado del paciente diabético, promueve estilos de vida saludables, mejorando la calidad de vida saludable contribuyendo así a disminuir las altas tasas de morbimortalidad de la diabetes, especialmente amputación de miembros inferiores La educación para el paciente diabético sigue siendo una herramienta fundamental para el control de la diabetes Mellitus, cualquier diferencia en la dieta, sedentarismo, stress, elevación de los triglicéridos, estilos de vida desfavorables u otros factores de riesgo afecta directamente al paciente con diabetes. Se estima que con un cuidado correcto de los pies, se podría prescindir de un $75 \%$ de todas las amputaciones en personas con diabetes mellitus (3).

En estudio internacional, en Venezuela Varela Alva G. et. al., realizaron un estudio con el objeto de evaluar el auto cuidado de los pies en pacientes con diabetes mellitus tipo 2 en la consulta de Medicina de Familia del Hospital I Tulio Febres Cordero de La Azulita, Se trata de una Investigación de campo y diseño cuasi experimental con pre test y pos-test. Participaron 100 pacientes con diagnóstico de 
diabetes mellitus tipo 2 . El $74,0 \%$ reciben hipoglucemiantes orales. La mayoría negó antecedentes de úlceras en los pies. Se evidenció callosidades, deformidades y onicopatías, categorizándose como pie de riesgo aumentado, después de la intervención hubo una disminución significativa. Los conocimientos y hábitos de auto cuidado fueron deficientes antes de la intervención educativa, después el cambio fue altamente significativo $(p<0,001)$ para casi todos los riesgos. En conclusión el auto cuidado en los pacientes mejoró significativamente, demostrando el beneficio de la educación para la salud en la atención primaria aplicada por el Médico de Familia (4).

Estudio Nacional en Trujillo, por Farfán Sánchez, Rosa "Efectividad de un programa educativo basado en el auto cuidado de miembros inferiores para la prevención de complicaciones en pacientes diabéticos Hospital de Sullana. 2007" tuvo como objetivo central el establecer si un programa educativo basado en el auto cuidado de miembros inferiores seria efectivo para la prevención de complicaciones en pacientes diabéticos del Hospital de Sullana Trujillo Concluye: Los pacientes diabético que no llevaron el programa educativo presentan alto grado de lesión y complicaciones; mientras que los el grupo experimental aumentaron su nivel de conocimiento sobre evitar complicaciones de la diabetes.

.El programa educativo aplicado al grupo experimental permitió comprobar su efectividad en el objetivo de lograr un mejor auto cuidado de miembros inferiores para evitar complicaciones en el pie diabético Los pacientes del grupo control, si bien no mejoraron su nivel de lesiones, al aplicárseles el programa de manera alternada respondieron con una mejor predisposición a mejorar su auto cuidado y evitar las complicaciones en sus miembros inferiores (5).
El presente estudio realizado se justifica porque proporciona datos importantes a la institución para que a través de las personas encargadas de la estrategia consideren las acciones preventivas promocionales considerando la educación permanente como herramienta fundamental en la disminución de complicaciones de miembros inferiores en el paciente diabético. Tuvo como objetivo evaluar la efectividad del programa educativo sobre autocuidado en miembros inferiores de los pacientes con diabetes mellitus 2 que acuden al Hospital Santa María del Socorro de Ica

\section{MATERIAL Y METODOS}

El estudio fue una investigación cuantitativa, aplicativa, Explicativa ,longitudinal, con diseño: Cuasi experimental, comparativo, con grupo control y grupo experimental con pre test y post test, ejecutándose en el Hospital Santa María del Socorro de Ica Perú , en el servicio de medicina hospitalización, la muestra estuvo constituido de 100 pacientes utilizándose el muestreo no Probabilístico intencionado,50 para grupo control y 50 para grupo experimental que reunieron los criterios de selección y consentimiento informado seguido de un programa educativo de secciones $\mathrm{La}$ validez se determinó mediante juicios de expertos se hizo uso del coeficiente y la confiabilidad, alfa de Cronbach fue de 0,79, la técnica de análisis e interpretación de datos fue debidamente codificada e ingresada en una base de datos utilizando el paquete estadístico SPSS Versión 14,0 para su procesamiento, luego se procedió a elaborar las tablas respectivas para atender a los objetivos trazados

\section{RESULTADOS}

Antes del programa educativo (pre- test), el nivel de conocimiento sobre del pie diabético en pacientes con diabetes mellitus tipo 2 (sobre el cambio del color de la piel, ejercicios físicos para prevenir el pie diabético, perdida de la sensibilidad, uso de cremas para evitar 
resequedad de la piel, riesgo de uñas encarnadas) , era bajo, tanto en el grupo control $(37,6 \%)$ como en el grupo experimental $(30,4 \%)$.

Después de aplicado el programa educativo (post test), el nivel de conocimiento en el grupo experimental alcanzo un buen nivel de conocimiento $(71,6 \%)$, en cambio el nivel de conocimiento en el grupo control continuo siendo bajo $(37,6 \%)$

Antes del programa educativo (pre test), el nivel de auto cuidado de miembros inferiores por pacientes con diabetes mellitus tipo 2 (estilos de vida, auto cuidado en los pies, de la uñas, higiene de los pies y con el calzado) es inadecuado, tanto en el grupo control $(29,28$ $\%)$ como en el grupo experimental $(30,64 \%)$ Después del programa educativo (pos test), el grupo experimental obtuvo un nivel de auto cuidado adecuado de $(75,88 \%)$, el grupo control continuo siendo inadecuado $(29,28 \%)$ al no recibir el programa educativo. Posteriormente se aplicó el programa educativo al grupo control

Se demostró que los pacientes lograron incrementar sus conocimiento sobre autocuidado de miembros inferiores (estilos de vida, cuidados de pies, autocuidado con las uñas, sobre higiene de los miembros inferiores, y el cuidado con el uso del calzado) y en la prevención de complicaciones para evitar la amputación de los miembros inferiores. en los pacientes de estudio

El programa educativo sobre auto cuidado en la prevención de complicaciones de miembros inferiores (pie diabético) es efectivo porque incrementa el nivel de conocimiento y mejora las actitudes en el auto cuidado.
TABLA N 1

\begin{tabular}{lcccc}
\hline \multicolumn{5}{c}{ GRUPO } \\
CONTROL & EXPERIMENTAL \\
CARACTERISTICAS & & & \\
& N & $\%$ & N & $\%$ \\
EDAD & & & & \\
$30-40$ a & 02 & 08 & 03 & 12 \\
$41-50$ a & 07 & 28 & 03 & 12 \\
$51-60$ a & 09 & 36 & 11 & 44 \\
& & & & \\
$61-70$ a & 05 & 20 & 05 & 20 \\
71 a + & 02 & 08 & 03 & 12 \\
SEXO & & & & \\
Femenino & 17 & 68 & 18 & 72 \\
Masculino & 08 & 32 & 07 & 28 \\
& & & & \\
EDUCACIÓN & & & & \\
Primaria & 02 & 08 & 03 & 12 \\
Secundaria & 17 & 68 & 16 & 64 \\
Superior Tec. & 03 & 12 & 04 & 16 \\
Superior Univ. & 03 & 12 & 02 & 08 \\
\hline
\end{tabular}

TABLA $\mathbf{N}^{\circ} 2$

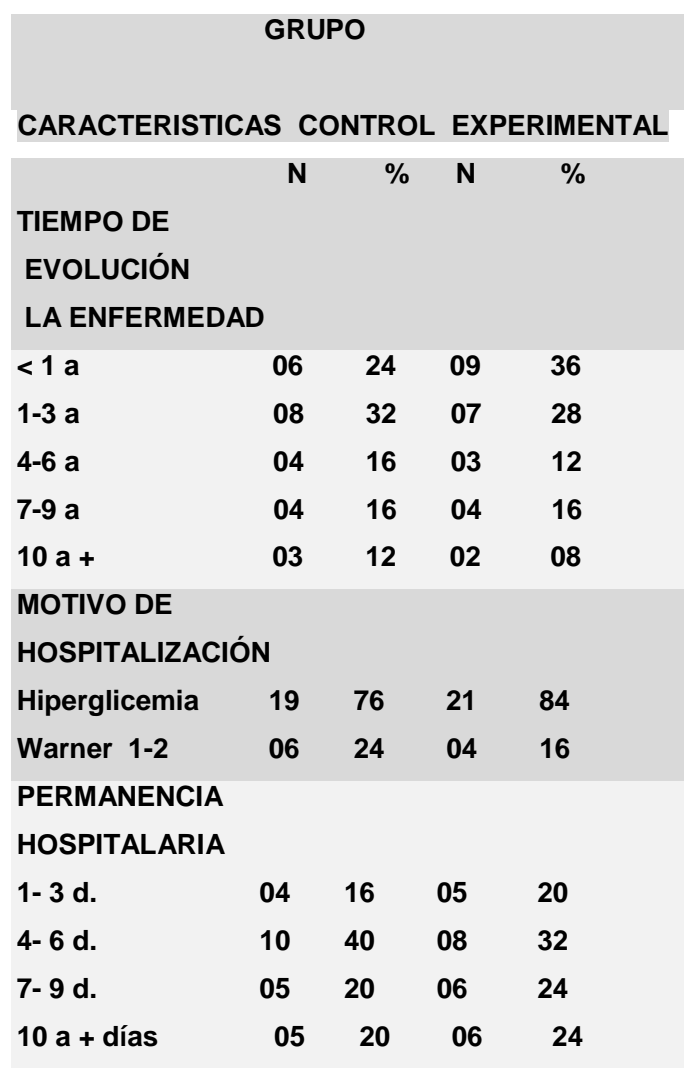


TABLA N 3. PROMEDIO DE VARIABLES DE ESTUDIO DE USUARIOS CON DIABETES MELLITUSTIPO 2 PRE -TEST

\begin{tabular}{|c|c|c|c|c|c|c|c|c|}
\hline \multirow[t]{4}{*}{ PROMEDIO TOTAL } & \multirow{2}{*}{\multicolumn{4}{|c|}{ CONTROL }} & \multirow{2}{*}{\multicolumn{4}{|c|}{ EXPERIMENTAL }} \\
\hline & & & & & & & & \\
\hline & \multicolumn{2}{|l|}{ SI } & \multicolumn{2}{|l|}{ NO } & \multicolumn{2}{|l|}{ SI } & \multicolumn{2}{|l|}{ NO } \\
\hline & $\mathbf{N}$ & $\%$ & $\mathbf{N}$ & $\%$ & $\mathbf{N}$ & $\%$ & NO & $\%$ \\
\hline CONOCIMIENTO & 9,4 & 37,6 & 15,6 & 62,4 & 7,6 & 30,4 & 17,4 & 69,4 \\
\hline TOTAL & 9,6 & 37,6 & 15,6 & 62,4 & 7,6 & 30,4 & 17,4 & 69,4 \\
\hline AUTOCUIDADO : ESTILOS DE VIDA & 7 & 28 & 18 & 72 & 7,6 & 30,4 & 17,4 & 69,6 \\
\hline AUTOCUIDADO : EN PIES & 9,5 & 38 & 15,5 & 62 & 15,5 & 34,8 & 9,5 & 65,2 \\
\hline AUTOCUIDADO : DE UÑAS & 9,9 & 28 & 15,1 & 72 & 10,9 & 30,4 & 14,1 & 69,6 \\
\hline $\begin{array}{l}\text { AUTOCUIDADO : HIGIENE DE LOS } \\
\text { PIES }\end{array}$ & 7,8 & 31,2 & 17,2 & 68,8 & 8,1 & 32,4 & 16,9 & 67,6 \\
\hline AUTOCUIDADO : CON EL CALZADO & 5,3 & 21,2 & 19,7 & 78,8 & 6.3 & 25,2 & 18,7 & 74,8 \\
\hline TOTAL & 7,9 & 29,28 & 17,1 & 70,72 & 9,68 & 30,64 & 15,32 & 69,36 \\
\hline
\end{tabular}

TABLA N4 PROMEDIO DE VARIABLES DE ESTUDIO DE USUARIOS CON DIABETES MELLITU TIPO 2 POST- TEST

\begin{tabular}{|c|c|c|c|c|c|c|c|c|}
\hline \multirow{4}{*}{ PROMEDIO TOTAL } & \multicolumn{8}{|c|}{ POST- TEST } \\
\hline & \multicolumn{4}{|c|}{ CONTROL } & \multicolumn{4}{|c|}{ EXPERIMENTAL } \\
\hline & SI & & NO & & SI & & NO & \\
\hline & $\mathbf{N}$ & $\%$ & $\mathbf{N}$ & $\%$ & $\mathbf{N}$ & $\%$ & NO & $\%$ \\
\hline CONOCIMIENTO & 9,4 & 37,6 & 15,6 & 62,4 & 17,9 & 71,6 & 7,1 & 28,4 \\
\hline TOTAL & 9,6 & 37,6 & 15,6 & 58,4 & 17,9 & 71,6 & 7,1 & 28,4 \\
\hline AUTOCUIDADO : ESTILOS DE VIDA & 7 & 28 & 18 & 72 & 16,2 & 64,8 & 8,8 & 35,2 \\
\hline AUTOCUIDADO : EN PIES & 9,5 & 38 & 15,5 & 62 & 19,8 & 79,2 & 5,2 & 20,8 \\
\hline AUTOCUIDADO : DE UÑAS & 9,9 & 28 & 15,1 & 72 & 18,7 & 75,8 & 6,3 & 24,2 \\
\hline $\begin{array}{l}\text { AUTOCUIDADO : HIGIENE DE LOS } \\
\text { PIES }\end{array}$ & 7,8 & 31,2 & 17,2 & 68,8 & 21,6 & 86,4 & 3,4 & 13,6 \\
\hline AUTOCUIDADO : CON EL CALZADO & 5,3 & 21,2 & 19,7 & 78,8 & 18,3 & 73,2 & 6,7 & 26,8 \\
\hline TOTAL & 7,9 & 29,28 & 17,1 & 70,72 & 18,92 & 75,88 & 6,08 & 24,12 \\
\hline
\end{tabular}

\section{DISCUSIÓN}

El pie diabético es una de las complicaciones de la diabetes mellitus tipo 2, que se incrementa año por año con etiología multifactorial, teniendo como factores principales la infección, isquemia neuropatía, tiene características clínicas frecuentes como ulceras gangrena y amputación en las extremidades inferiores ocasionando en el paciente discapacidad aislamiento.
De acuerdo con los resultados obtenidos en el trabajo en relación a la efectividad de un programa educativo sobre auto cuidado en la prevención de complicaciones de miembros inferiores (pie diabético) en el hospital Santa María del Socorro, al aplicar el pre- tés a los pacientes en estudio, grupo control y grupo experimental ,nos nuestra que el nivel de conocimiento sobre prevención de complicaciones en miembros inferiores (pie 
diabético) es bajo en el grupo control con $37,6 \%$ al igual se encuentra en el grupo experimental con un nivel de conocimiento bajo de $30,4 \%$ tabla $N^{\circ} 3$ Similares resultados se encontraron en un estudio de JIMENÉS ESTRADA, Georgina; Martínez Borroso, María Teresa; GOMÉZ Arcilla, Martha "Nivel de conocimiento del paciente diabético sobre la prevención del pie diabético" Concluye: El nivel de conocimiento en la prevención del pie diabético en personas con Diabetes Mellitus tipo 2 predomina el nivel de conocimiento bajo, seguido(41\%) (6). Otro estudio por CABRERA MORON, MOTTA QUIJANDRIA IRMA, Rodríguez Robledillo Cynthia, VELASQUEZ Carranza "Nivel de conocimiento sobre autocuidado en la prevención de complicaciones Diabéticas en usuarios del policlínico Chincha Es salud 2009, concluye el 30,0 \% con conocimiento bajo y $15,3 \%$ conocimiento alto; con respeto al auto cuidado en la prevención de complicaciones diabéticas (7).

En cuanto al auto cuidado de los miembros inferiores en los pacientes (sobre estilos de vida, auto cuidado de los pies de las uñas, higiene de los pies y el calzado ) se aplicó el pre test al grupo control dando como resultado como inadecuado en el grupo control con un ( $70,72 \%$ )y el $(69.36 \%)$ en el grupo experimental. similares resultados se encontró en un estudio GUILLERMO ENRIQUE Castellanos I Trabajo Especial "Evaluación de riesgo para desarrollo de pie Diabético en sujetos con Diabetes Mellitus Tipo 2" República Bolivariana de Venezuela 2011 quien concluye: es alto el porcentaje de diabéticos con riesgo de padecer pie diabético, siendo este riesgo mayor en el hombre(8).

En relación al post-tés después del programa educativo el nivel de conocimiento mejoro en el grupo experimental con $71,6 \%$, mientras en el grupo control continuo con el mismo porcentaje de $37,6 \%$ del pre- tés, tabla $N^{\circ} 4$ similares resultados se encontró en un estudio de FARFAN SÁNCHEZ Rosa." Efectividad de un programa educativo basado en el auto cuidado de miembros inferiores para la prevención de complicaciones en pacientes diabético" hospital de Sullana 2007 (3). El programa educativo aplicado al grupo experimental permitió comprobar su efectividad en el objetivo de lograr un mejor auto cuidado de miembros inferiores para evitar complicaciones en el pie diabético Al grupo control, al aplicárseles el programa de manera alternada respondieron a una mejor predisposición a mejorar su auto cuidado y evitar las complicaciones en sus miembros inferiores Otro estudio por TEJEDOR HERNANDEZ Lucia. "Prevención del pie Diabético a través del auto cuidado" Universidad de Zaragoza Concluye refiriendo es fundamental promover hábitos de auto cuidado de los pies y de la diabetes en general y concienciar de la importancia que tiene el realizarlos de una manera correcta, ya que con un buen programa educativo se pueden llegar a prevenir el $85 \%$ de las amputaciones, una de las peores consecuencias del pie diabético (9).

\section{Correspondencia:}

Mag. Flora Martha Huisacayna Díaz

Correo electrónico: martha.f.h.d@gmail.com

\section{REFERENCIAS BIBLIOGRAFICAS}

1. Organización Mundial de la Salud. (OMS) Centro de Prensa, Nota Descriptiva No.312,2010 (Internet) OMS;2015 ( citado el 12 de noviembre 2015 Disponible en: http://www.who.int/mediacentre/factsheets/f s3312/es/ index.hom/.

2. Hospital Universitario Ramón y Cajal. Dirección de Enfermería Salud Madrid Comunidad de Madrid Protocolo de cuidados PIE DIABETICO PRT7PD/001 Junio 2005 p. 3

3. Farfán S. Efectividad de un programa educativo basado en autocuidado de miembros inferiores para la prevención de complicaciones en pacientes diabéticos Tesis. Trujillo, Perú. Facultad de Enfermería, Universidad Nacional de Trujillo; 2007

4. Varela A, Mejía M, D'Ávila C. Autocuidado de los pies en pacientes Diabéticos tipo 2 
.Universidad de los Andes Mérida Venezuela Feb.2015 Rev. Médico de Familia. 2015; 23 (1)

5. Farfán S. Efectividad de un programa educativo basado en el auto cuidado de miembros inferiores para la prevención de complicaciones en pacientes diabéticos hospital de Sullana, 2007. Tesis Segunda especialidad. Piura, Sullana, Perú Facultad de Enfermería, Universidad Nacional de Trujillo; 2007

6. Jiménez E, Martínez B, Gómez A. Nivel de conocimiento del paciente diabético sobre la prevención del pie diabético. Tesis Pregrado. Lima, Perú. Facultad de Medicina, EAP Enfermería, Universidad Nacional Mayor de San Marcos; 2015;
7. Cabrera M, Motta Q, Rodríguez R, Velázquez $\mathbf{C}$. Nivel de conocimiento sobre autocuidado en la prevención de complicaciones diabéticas en usuarios del policlínico Chincha - Es Salud - 2009 "Rev. Enfermen Heredia 2010"

8. Castellanos G. Trabajo Especial Evaluación de riesgo para desarrollo de pie diabético en sujetos con diabetes mellitus tipo 2 Republica Boliviana de Venezuela; 2011

9. Tejedor P. Prevención del Pie Diabético a través del auto cuidado. Tesis. Zaragoza, España. Universidad de Zaragoza.

Recibido: 20/07/ 2016

Aprobado para Publicación: 30/10/2016 